[Agr. Biol. Chem., Vol. 29, No. 8, p. 714 718, 1965]

\title{
Phytochemical Studies on the Tobacco Alkaloids
}

\section{Part VIII. Biosynthesis of the Pyrrolidine Ring of Nornicotine in the Excised Root Culture}

\author{
By Shigenobu Mizusakr, Takuro Kisakr and Einosuke Tamaki \\ Central Research Institute, Japan Monopoly Corporation, 1-28.3, \\ Nishishinagawa, Shinagawa-ku, Tokyo, Japan \\ Received January 18, 1965
}

\begin{abstract}
Duplicate feeding experiments of $d l$-ornithine-2-14 $\mathrm{C}$ to the excised tobacco root culture were made, and the radioactive nornicotine was isolated. Approximately two thirds of the radioactivity was located in the 2-position of the pyrrolidine of the nornicotine in these experiments. This fact indicates that there are two modes in nornicotine biosynthesis: exclusive incorporation to the $\mathrm{C}-2$ and equal incorporation to $\mathrm{C}-2$ and $\mathrm{C}-5$ from $\mathrm{C}-2$ of ornithine.
\end{abstract}

On the basis of this finding, biosynthetic route was discussed.

\section{INTRODUCTION}

It has been well established that nicotine is synthesized in tobacco roots and that nornicotine is produced in the leaves by demethylation of nicotine which has been accumulated there by transpiration. ${ }^{1 \prime}$ Recent studies on the alkaloid biosynthesis using isotopically labeld compounds have shown that ornithine$2-{ }^{14} \mathrm{C}^{2,3)}$ and certain other amino acids of the glutamic acid family ${ }^{4 \sim 6)}$ are incorporated into nicotine to give the activity equally on $\mathrm{C}-2$ and C-5 of the pyrrolidine ring. For the explanation of the equal labeling on C-2 and C5 , Leete ${ }^{7)}$ has proposed a biosynthetic scheme for nicotine, in which nornicotine is firstly synthesized via a symmetric mesomeric anion of $A^{1}$-pyrroline derived from those amino acids

1) R. F. Dawson, Advances in Enzymol., 8, 203 (1948).

2) E. Leete and K.J. Siegfried, J. Am. Chem. Soc., 79, 4529 (1957).

3) B.L. Lamberts, L. J. Dewey and R. U. Byerrum, Biochim. Biophys. Acta, 33, 22 (1959).

4) E. Leete, I. Am. Chem. Soc., 80, 2162 (1958).

5) B. L. Lamberts and R. U. Byerrum, I. Biol. Chem., 233, 939 (1958).

6) E. Leete, E. G. Gros and T. J. Gilbertson, Tetrahedron Letiers, 587 (1964).

7) E. Leete; "Biogenesis of Natural Compounds" Perga. mon press, p. 739 (1963). and then methylation takes place to give nicotine. Ladesic and $\mathrm{Tso}^{8)}$ have also reported that nornicotine can be a precursor of nicotine in the plant at an early stage of the growth. Consequently, it is most likely that nornicotine, in certain cases, is produced in tobacco plants independently of nicotine and serves as a precursor of nicotine.

According to our results reported in a series of studies ${ }^{9,10)}$ on the tobacco alkaloids, however, it seems to be inconceivable that nornicotine, being always optically impure in the plant, can be methylated to give nicotine which is always the optically pure $l$-form in the plant. Recently we have reported that nicotine can give nornicotine by demethylation not only in the leaf but also in the root. The presence of nornicotine in the root, a locus of the nicotine synthesis, has often been thought to support nornicotine as a precursor of nicotine, but this result may cast doubt on such inference. At this point it seems to be

8) Ladesic and T.C. Tso, Phytochemistry, 3, 541 (1964),

9) T. Kisaki and E. Tamaki, Arch. Biochem. Biophys. 92, 351 (1961).

10) T. Kisaki and E. Tamaki, Naturwissenschafien, 47, $450(1960)$. 
noteworthy to point out the study ${ }^{11)}$ of Leete on the anabasine biosynthesis. He has reported that anabasine, a secondary amine alkaloid like nornicotine, is synthesized from lysine in Nicotiana glauca in such a way as the $\alpha$-carbon of lysine is incorporated only into the carbon-2 of the piperidine ring and has concluded that the biosynthetic mode of anabasine from lysine is different from that of nicotine from ornithine.

There has been no report on the distribution of radioactivity of nornicotine which is in most cases accompanied with nicotine in the tobacco root. As cited above, it has been well established that radioactive carbon on the 2-position of ornithine is incorporated into both $\mathrm{C}-2$ and $\mathrm{C}-5$ of the pyrrolidine ring of nicotine in equal amounts. Then, if nicotine can be derived from nornicotine by methylation in the root, administration of ornithine${ }_{2}^{2}{ }^{14} \mathrm{C}$ results in nornicotine having equally labeled pyrrolidine ring on $\mathrm{C}-2$ and $\mathrm{C}-5$.

Such a study seemed to be of some interest to clarify the biogenetic relationship between nicotine and nornicotine. For this reason, ornithine-2- ${ }^{14} \mathrm{C}$ was administered aseptic cultures of excised tabacco roots and both nicotine and nornicotine isolated from the cultures were analysed for their radioactivity.

\section{EXPERIMENTAL}

\section{Excised root culture}

Following the method of Solt, ${ }^{12}$ ) excised roots of N. rustica, var. Brasilia were cultured aseptically at $29^{\circ} \mathrm{C}$.

\section{Tracer}

$d l$-Ornithine-2-14C (specific acitivity, $26 \mathrm{mc} / \mathrm{mM}$ ) was purchased from California Corp. for Biochem. Res., U. S. A.

\section{Feeding of dl-ornithine-2-14 $\mathrm{C}$}

Duplicate experiments were carried out. In general, the cultures were incubated for one week prior to the addition of the tracer. The tracer was sterilized by autoclaving and one $\mu \mathrm{c}$ was pipetted aseptically to each excised root culture. In Experiment 1, 40 cultures were employed and incubation was continued

11) E. Leete, Am. J. Chem. Soc., 78, 3520 (1956).

12) M. L. Solt, Plant Physiol., 32, 480 (1957). for an additional 30 days. In Experiment 2, 100 cultures were employed and incubation was continued for an additional 24 days.

\section{Isolation of alkaloids}

After the incubation periods, the cultures of each experiment were harvested by separating root mass from spent culture fluids through a fritted glass filter. The root mass was rinsed throughly with water. The washings were combined with the spent culture fluids. The root mass was macerated in Waring blender with $50 \mathrm{ml}$ of $75 \%$ ethanol containing $0.2 \%$ acetic acid and filtered through a fritted glass filter. The remaining mass on the funnel was extracted five times with the same volume of the same solvent. The extracts were combined and the volume was reduced in vacuo. Insoluble matter formed during the concentration was removed by centrifugation. The supernatant was allowed to pass through a column $(2 \times 10 \mathrm{~cm})$ of Dowex $50 \times 4\left(\mathrm{H}^{+}\right)$. The column was washed with a sufficient volume of water and eluted with $250 \mathrm{ml}$ of $2 \mathrm{~N}$ ammonia water which served to remove alkaloids and amino acids. The alkaloids were extracted from the eluate by shaking five times with $50 \mathrm{ml}$ of chloroform. The chloroform extracts were combined and dried over anhydrous sodium sulfate.

On shaking the chloroform extract with $30 \mathrm{ml}$ of $\mathrm{N}$ hydrochloric acid three times, the alkaloids were tranferred into the acidic aqueous solution. The acidic aqueous solution was reduced to dryness under diminished pressure, and employed for paper chromatography and autoradiography of the alkaloids. The eluate which had been extracted with chloroform was employed for paper chromatography and radioautography of the amino acids after concentration.

The spent culture fluids were acidified with acetic acid and were reduced to $10 \mathrm{ml}$ in vacuo. After addition of $l$-nicotine and $l$-nornicotine, $300 \mathrm{mg}$ each, to the solution as carrier, the alkaloids in the solution were continuously extracted with chloroform after alkalinization for 24 hours. The chloroform extract was dried over anhydrous sodium sulfate and, on evaporation of the solvent, the residue was subjected to liquid chromatography on an alumina column in the same manner as described previously.9) Nicotine was eluted with ether and nornicotine was eluted with $3 \%$ methanol in ether. The alkaloid in the respective eluates was transferred to the hydrochloric acid solution by shaking the eluate with $\mathrm{N}$ hydrochloric acid solution. After removal of some impuri- 
ties in the solutions by several extractions with ether at $\mathrm{pH}=5.0$, both solutions were made alkaline and continuously extracted with chloroform. The chloroform extracts were treated with $\mathrm{N}$ hydrochloric acid solution in the same way as above. The acidic solutions were concentrated to dryness in vacuo and each alkaloid was converted to its corresponding picrate. Five recrystallizations of the resultant picrates gave nicotine dipicrate, m. p. $222 \sim 223^{\circ} \mathrm{G}$ and nornicotine dipicrate, m.p. $191 \sim 192^{\circ} \mathrm{C}$, both with constant radioactivity.

\section{Degradation of nicotine and nornicotine}

Nicotine dipicrate was converted to the hydrochloride and was degraded with $\mathrm{KMnO}_{4}$ to give nicotinic acid, according to the method described by Dawson et al. ${ }^{13)}$ Nornicotine dipicrate was decomposed with $2.5 \mathrm{~N}$ hydrochloric acid and the resultant free picric acid was removed by extraction with ether. The hydrochloric acid solution was reduced to dryness in vacuo. To the residue, $1 \mathrm{ml}$ of $70 \%$ perchloric acid was added and the perchlorate of nornicotine was formed on standing in a vacuum desiccator. The perchlorate was recrystallized from methanol-ether and its radioactivity was assayed.14) The crystalline perchlorate and the mother liquor were again combined and oxidatively degraded to nicotinic acid in the same manner as in the nicotine degradation.

Following the Weidel's procedure, ${ }^{15)}$ the nicotinic adid derived from either nicotine or nornicotine was decarboxylated to pyridine, which was then converted to its picrate. Both picrates were recrystallized separately from water.

\section{Assay of radioactivity}

The radioactivity of all the solid samples was measured at infinite thickness by end window gasflow counter, Aloka Fc-14 and corrected for self-absorption. The liquid samples were dried on planchets and measured by windowless gasflow counter, Hitachi RDF-4.

\section{Paper chromatography and autoradiography}

The alkaloid fraction was spotted on Whatman No. 1 paper, which was pre-impregnated with $0.2 \mathrm{~N}$ acetate and was developed with the upper layer of tert-amyl alcohol : $0.2 \mathrm{~N}$ acetate buffer $(\mathrm{pH}=5.6)=1: 1$. The amino acid fraction was paper-chromatographed

13) R. F. Dawson, D, R. Christman, A. D'Adamo, M. L. Solt and A. P. Wolf, Am. J. Chem. Soc., 82, 2628 (1960).

14) The perchlorate had about a half radioactivity of the pictrate, indicating that the nornicotine obtained is a racemic form.

15) H. Weidel, Ann., 165, 328 (1873). on Whatman No. 1 paper according to the method described by Levy ${ }^{16)}$; butanol: acetic acid: water $=$ $4: 1: 5$ in the first dimension, $m$-cresol:phenol: borate buffer $(\mathrm{pH}=8.3)=4: 2: 1$ in the second dimension.

The autoradiogram was made on a Sakura X-ray film (Industrial Type N) for 20 to 30 days.

\section{RESULTS AND DISCUSSION}

On a paper chromatogram of the alkaloid fraction obtained from the excised root cultures fed with $d l$-ornithine-2- ${ }^{14} \mathrm{C}$ for 24 days, it was observed that there were nornicotine, anabasine, anatabine and several other Koenigpositive substances as minor alkaloids in addition to nicotine.

As shown in Fig. 1, only negligible radioactivity was found in anatabine $(\mathrm{C})$ and an-

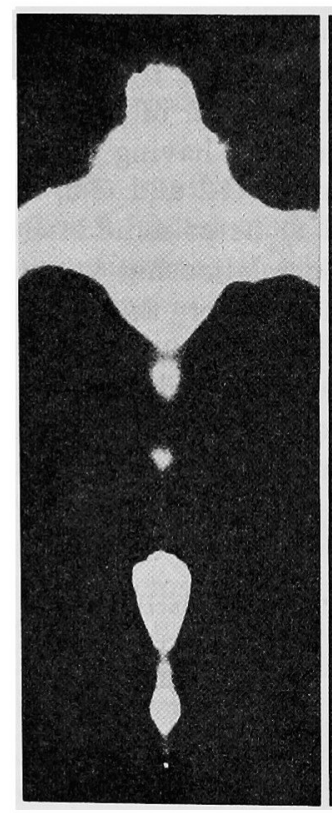

(a)

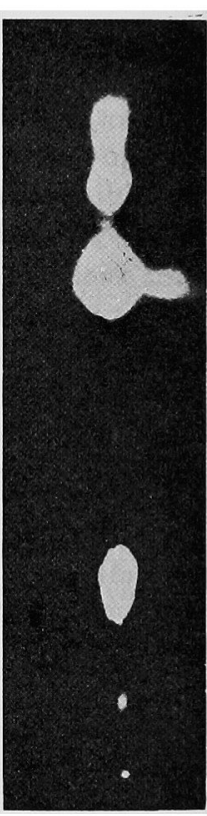

(b)

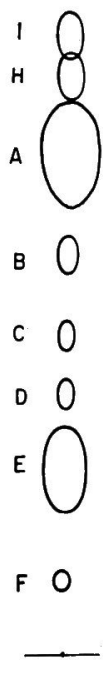

(c)
FIG. 1. Autoradiogram of the Alkaloid Fraction.

(a) and (b) are the autoradiograms of the alkaloid fraction and (c) is the paper chromatogram, the color of which was developed with $p$-aminobenzoic acid and $\mathrm{BrCN}$ vapor.

(a) was spotted under cool air blow and (b) was spotted under hot air blow.

A, Nicotine B, Unknown C, Anatabine D, Anabasine E, Nornicotine F, Nicotinic acid(?) H, I, Unknown

16) A. L. Levy and P. Chung, Anal. Chem., 25, 396 (1953). 
abasine (D) whereas significant activity was located not only in nicotine, but also in nornicotine (E) and three other minor alkaloids, $\mathrm{F}, \mathrm{H}$ and $\mathrm{I}$. The latter three minor alkalids are not yet identified, but $\mathrm{H}$ and $\mathrm{I}$ were extractable with ether at $\mathrm{pH}=5$.

Table I shows the distribution of the radioactivity in the major fractions. Radioactivity

TABLE I. DISTRIBUTION OF RADIOACTIVITY IN VARIOUS FRACTIONS

\section{Fraction}

Culture fluid

$75 \%$ Ethanol-soluble fraction of the roots

Chloroform extract of the culture fluids

Nicotine fraction

Nornicotine fraction

These are the data in Expt. 2.

TABLE 11. DISTRIBUTION OF ${ }^{14} \mathrm{C}$ IN NiCOTINE FROM The Excised Tobacco Root Culture Fed WITH $d l$-ORNITHINE-2-14C

\section{Compound}

Expt. 1 (30 days)

Nicotine dipicrate

Nicotinic acid picrate

Nicotinic acid/nicotine

Expt. 2 (24 days)

Nicotine dipicrate

Nicotinic acid

Pyridine picrate

Nicotinic acid/nicotine

TABLE III. Distribution of ${ }^{14} \mathrm{C}$ IN NoRnicotine From THE ExcISED TOBaCco RoOT CuLTuRE FED WITH $d l$-ORNITHINE-2-14 C

\section{Compound}

Specific activity Ratio of $\begin{array}{ll}\text { c.p.m. } / \mathrm{mM} \times 10^{-7} & \text { specific } \\ \text { activity }\end{array}$

Expt. 1 (30 days)

Nornicotine dipicrate

4.9

Nicotinic acid picrate

3.4

Nicotinic acid/nornicotine

Expt. 2 (24 days)

Nornicotine dipicrate

Nicotinic acid

Pyridine picrate

Nicotinic acid/nornicotine
Total activity $\times 10^{7}$ (c.p.m.)

2.72

0.58

0.34

0.26

0.02
5 times as high as that in the $75 \%$ ethanol extract from the root mass was found in the culture fluids. Alkaloidal costitution in both the root mass and culture solutions was proved to be identical on a paper chromatogram. The nicotine and nornicotine fractions after separation on alumina column contained 75 and $6 \%$ of the radioactivity of the alkaloid fraction, respectively. However, the nicotine was still contaminated with a minute quantity of nornicotine. Hence, the overall radioactivity of nornicotine should be more than $6 \%$.

Distribution of the radioactivity in the nicotine and nornicotine molecules in duplicate experiments is shown in Tables II and III. With nicotine, half the activity was located in the 2-position of the pyrrolidine ring, but with nornicotine $68 \%$ (average value of the duplicate experiments) of the radioactivity was in the 2-position. The pyridine rings of the nicotine and nornicotine contained only negligible radioactivity. It has been proved several workers ${ }^{2,3}$ that the activity of ornithine- $2-{ }^{14} \mathrm{C}$ is incorporated equally at $\mathrm{C}-2$ and $\mathrm{C}-5$ of the pyrrolidine ring of nicotine. On the basis of these facts, it might be deduced that the remaining radioactivity other than that being at $\mathrm{C}-2$ of the pyrrolidine ring of nornicotine is presumed to be located at $\mathrm{C}-5$. If so, the radioactivity of the nornicotine at $\mathrm{C}-2$ and $\mathrm{C}-5$ is roughly in a ratio of $2: 1$.

An explanation for the unequality of the radioactive distribution in the nornicotine is that there are at least two types of nornicotine; one is presumably equally labelled at C-2 and $\mathrm{C}-5$ in the same mode as with nicotine and the other is only radioactive at C-2. Speculating from the radioactive distribution in the nornicotine, the ratio of the biogenetic route for the equally labeled nornicotine to that for the $\mathrm{C}-2$ active nornicotine is about $2: 1$. In previous report ${ }^{17)}$ from our laboratory, it

$$
\begin{aligned}
& 3.6 \\
& 2.4 \\
& 0.03
\end{aligned}
$$

17) S. Mizusaki, T. Kisaki and E. Tamaki, This Journal, $29,78(1965)$. 
was demonstrated that the tobacco root has an ability to convert nicotine to nornicotine as does the leaf. Accordingly, nornicotine derived from nicotine, which is labeled equally at C-2 and C-5, should also have equal radioactivity at $\mathrm{C}-2$ and $\mathrm{C}-5$. In this case, the nornicotine would not be a precursor of nicotine, but is a transformation product of nicotine. The nornicotine obtained in the present experiments should be this type of nornicotine. The metabolic route which gives the other type of nornicotine labeled only at $\mathrm{C}-2$ from ornithine- $2-{ }^{14} \mathrm{C}$ is unknown. Similar evidence has been demonstrated with the anabasine biosynthesis by Leete. ${ }^{11)}$ He reported that radioactivity of lysine- $2-{ }^{14} \mathrm{C}$ fed to $N$. glauca was solely located at the 2-position of the piperidine ring of anabasine which is also a secondary amine as is nornicotine. A similar explanation to that for the anabasine biosynthesis may be applied to the nornicotine which is radioactive only at $\mathrm{C}-2$. The biosynthesis of its pyrrolidine ring from ornithine is obviously different from the biosynthesis of the pyrrolidine ring of nicotine. According to Leete et al.'s scheme, ${ }^{6)}$ the $\alpha$-amino group of ornithine is deaminated to $\alpha$-keto- $\delta$-amino valeric acid, which then cyclizes to give 41 -pyrroline-2-carboxylic acid. Decarboxylation of the intermediate of the pyrrolidine ring proceeds before the conjugtion of the moiety to the pyridine ring and the intermediate produces a mesomeric anion of $\Delta_{1}$-pyrroline which gives nicotine with ${ }^{14} \mathrm{G}$ equally on the 2 - and 5 positions. Therefore, in the case of the nornicotine having the radioactivity solely at $\mathrm{C}-2$, the omission of the carboxyl originally from ornithine seems to occur after the formation of a bond between the incipient pyridine ring and the 2-position of precursor of the pyrrolidine ring. However, the nornicotine being active only at $\mathrm{C}-2$ cannot be a precursor of nicotine, which is labeled equally at $\mathrm{C}-2$ and $\mathrm{C}-5$ of the pyrrolidine ring.

The results with isotopic studies done by those authors cited before and in particular by Tso and his group ${ }^{8)}$ seem to indicate that nornicotine is produced before nicotine in the plant, and our present results do not eliminate such possibility. However, it is very evident from the present results that not all the nornicotine present in the root cultures can serve as a precursor of nicotine, since about one third of the nornicotine isolated was labeled only at $\mathrm{C}-2$. It is also very clear that this one third of the nornicotine is not produced at the expense of nicotine but could come before nicotine, although this nornicotine cannot be a precursor of nicotine.

Acknowledgement The authors thank Misses Yoko Shiozawa and Yōko Tanabe for their co-operations in this work. 